TITLE:

\title{
Spiral Growth of Colloidal Gold and Moiré Fringe
}

AUTHOR(S):

Suito, Eiji; Uyeda, Natsu

\section{CITATION:}

Suito, Eiji ... [et al]. Spiral Growth of Colloidal Gold and Moiré Fringe. Bulletin of the Institute for Chemical Research, Kyoto University 1961, 39(3): 251-252

ISSUE DATE:

1961-07-31

URL:

http://hdl.handle.net/2433/75828

RIGHT: 


$$
E_{B}-R T \ln A=\Delta F^{*}-R T \ln K,
$$

where $\Delta F^{*}$ is the free energy of activation for flow and $K$ a constant which depends upon the shear stress, the temperature and the vibrational frequencies of the liquid molecules concerned. Though neithcr $\Delta F^{*}$ and $K$ can be evaluated directly, the left side of the relation (2) can be estimated experimentally and it may give the measure relative to $\Delta \mathrm{F}^{*}$. It was shown that the linear relations between $\log V_{i}$ and $1 / T$ shift according to values of $\left(E_{B}-R T \ln A\right)$ at a certain temperature which depends upon the composition of the asphalts used.

Effects of colloidally fine fillers on the flow properties of asphalts were observed. It was found that carbon black reduces $V_{B}$ remarkably in comparison with colloidal silica and calcium carbonate.

\section{Spiral Growth of Colloidal Gold and Moiré Fringe \\ Eiji Surto and Natsu UyEdA \\ Nature, 185, 453 (1960)}

Various growth spirals and related matters recently observed by electron microscopy on lamellar single crystals of gold, prepared colloid-chemically under definite conditions as discussed in previous papers.

A small hole exists at the centre of the spiral, from where the ridge line starts and, after passing on the outer perimeter of the crystal, come back to the initial starting point. The two spirals on both surfaces of a crystal are nearly symmetrical to each other in most of the present examples.

In the dark-field image, formed with the $(2 \overline{2} 0)$ reflexion there appeared nearly parallel thick moir'e fringes, the direction of which is again parallel to $[2 \overline{2} 0\rfloor$ axis of the crystal. This means that they were formed by the interaction of two electron beams reflected respectively by each $(2 \overline{2} 0)$ plane contained in two superposed steps.

When the cause of the fringe is supposed to be attributable only to the slight azimuthal rotation of steps, the angle of rotation $\alpha$ is given by an equation: $\alpha=$ $d_{2 \overline{2} 0} / D$, where $D$ and $d_{2 \overline{2} 0}$ are the spacing of the moiré fringe and the interplanar

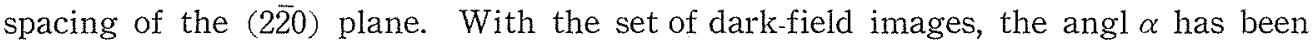
estimated to be of the order of $2 \times 10^{-3}$ red. on an average. Further, it increases roughly linearly from $7 \times 10^{-4}$ red. to $4 \times 10^{-3}$ red. or to much larger values as the position on the step, where the spacing of the fringe was meaured, approaches the centre of the crystal along the spiral step.

As for the small hole, Frank has derived the relationship between the hole diameter $\left(D_{0}\right)$ and the strength of the Burgers vector (b) at the core of the screw dislocation as follows, though slightly modified here:

$$
b=2 \pi\left(S \cdot D_{0} / G\right)^{1 / 2}
$$

where $S$ and $G$ are the surface free energy and modulus of rigidity of the material. The hole diameter is distributed over a range of $200-2400 \mathrm{~A}$. and the most 


\title{
ABSTRACTS
}

$1200 \mathrm{~A}$, while the mean value becomes $1020 \mathrm{~A}$. With this value, the stength of the Burgers vector was estimated to be $93-118 \mathrm{~A}$, where $2.76 \times 10^{11} \mathrm{dyne} / \mathrm{cm}^{2}$. and $600-$ $1,000 \mathrm{erg} / \mathrm{cm}$. were adopted for $G$ and $S$ respectively. On the other hand, we have also estimated the step height to be $109 \mathrm{~A}$ on an average by the shadow-casting method. This is of quite the same order as the strength of Burgers vector above obtained.

The Measurement of Particle Size of Ultra Fine Powders by the Air Permeability Method

\author{
Masafumi ArakawA and Eiji Surto \\ Kogyo Kagaku Zasshi (Journal of the Chemical Society of Japan, \\ Industrial Chemistry Section), 63, 556 (1960)
}

The particle sizes of ultra fine powders ranging from 20 to $400 \mathrm{~m} \mu$ have been measured by the air permeability of packed columns. As we leave the co-operative contributions of Poiseuille and Knudsen flows, two kinds of surface areas can be calculated by the application of a proper analytical method.

The experimental result suggests that the surface area deduced from the Poiseuille flow represents the geometric area of the secondary aggregated particles, while that deduced from the Knudsen flow is to be identified with the true surface area of the primary particles. The values of surface areas of various powders obtained from the Knudsen term agree with those calculated from electron microscopic data.

\section{Spiral Growth of Lamellar Single Crystal of Crystal of Colloidal Gold}

Eiji Surto and Natsu UYeDA

\author{
Journal of Electronmicroscopy, 8, 25 (1960)
}

The lamellar single crystal of colloidal gold, prepaed by the reduction of aqueous solution of auric chloride with salicylic acid at room temperature, sometimes shows a couple of growth spirals, which appear at the same time on two lamellar habit surfaces of a cystal. The three dimensional configuration of the crystal with spiral steps has been confirmed by the replica technique of the electron microscopy. A small hole can be observed at the centre of the spiral on most of the crystals having growth spirals. The equation presented by Frank giving the relationship between the Burgers vector and the diameter of such a hole has also been examined with respect to the crystal of colloidal gold.

A short discussion has also been made about the moiré fringes which appeared on the superimposed growth steps. 